PHARMACOGENETICS

\section{Misleading labels}

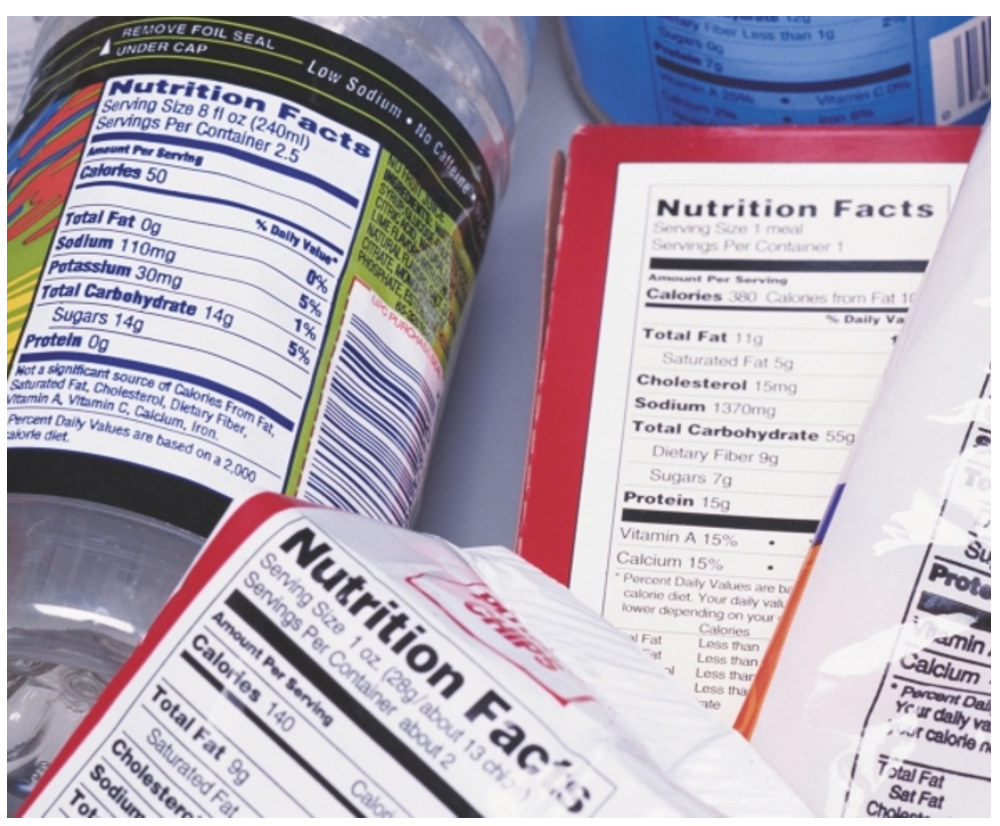

Ethnic labels, such as 'Black', 'Caucasian' and 'Asian', are frequently used to classify individuals in population-based studies. In drug trials, this classification has shown that drug response varies between certain ethnic groups. But is ethnicity the most informative label? If there is genetic variation that causes varied drug response within an ethnic group, combining trial results from a single ethnic group might obscure this variation. James Wilson and colleagues have investigated this issue by genetically classifying individuals, independently of their ethnicity, and then examining the probable significance of the genetic groupings. Strikingly, they show that genetic classification can uncover variation that would have been missed by ethnic classification, and that could have important consequences in drug response.

To classify individuals (from eight diverse ethnic groups), the authors genotyped them for 39 microsatellite markers. They then used a program called STRUCTURE to cluster individuals on the basis of the similarity of their genotypes, and four probable clusters were identified. Some ethnic groups were confined mostly to one cluster, but others fell into more than one. For example, only $24 \%$ of the Ethiopian group fell into the cluster that contains Black individuals (the ethnic group to which Ethiopians would normally be assigned), whereas $62 \%$ were grouped with Norwegians and Ashkenazi Jews.

Having defined the four genetic clusters, the authors then investigated their biological significance with respect to drug response. They did this by examining the frequencies of 11 functional variants in genes that are involved in drug metabolism. Sure enough, statistically significant differences were found in allele frequencies between some of the clusters. Importantly, some of these differences would have been missed if individuals had been classified according to standard ethnic labels. For example, the

\title{
Histone methylation takes the lead
}

How cells turn genes off and on has been puzzling geneticists and molecular biologists for many years. But a more-detailed picture is now emerging with recent insights into how two gene-silencing-associated events DNA methylation and histone modification - are linked. It began when methyl-binding proteins - which bind methylated cytosines in DNA - were discovered to associate with histone-modifying proteins that cause chromatin to adopt a transcriptionally repressive state. This link supported the idea that DNA methylation might cause gene silencing by influencing histone modification and chromatin remodelling leaving unanswered what controls DNA methylation itself. Now Tamaru and Selker report in Nature an exciting new finding that turns this idea on its head.

Unexpectedly, they have found that, in Neurospora, histone methylation controls DNA methylation, not vice versa.

Tamaru and Selker fortuitously generated the Neurospora dim-5 (defective in methylation 5) mutant, reported here, while trying to tag another dim gene. Once they had mapped dim-5, an obvious candidate gene came out of sequence searches in the region - a homologue of Drosophila Su(var) 3-9, which functions in heterochromatin formation and has recently been shown to methylate the Lys9 residue of histone (H)3. In complementation studies, this candidate gene rescued the methylation defects of dim-5 mutants. Furthemore, when wild-type dim- 5 was inhibited by quelling - a posttranscriptional form of gene silencing - it caused a methylated and silenced transgene to become demethylated and expressed.

The sequencing of this gene in dim-5 mutants revealed a single mutation that causes a premature stop codon in a SET domain, which can equip proteins with methyltransferase activity. And like two other SET-domain-containing proteins Su(var)3-9 and Clr4 (in Schizosaccharomyces pombe) - the dim- 5 candidate shows histone methyltransferase activity in vitro, its primary target being $\mathrm{H} 3$. But is it loss of this activity that causes defective DNA methylation? To show this, Tamaru and Selker transformed wild-type cells with a mutant form of $\mathrm{H} 3$ in which Lys9 was replaced by other amino acids. Transformed cells consistently showed loss of DNA methylation and the re-expression of a methylated transgene, indicating that $\mathrm{H} 3$ Lys9 is required for DNA methylation in Neurospora.

So how does this finding fit into the current picture of epigenetic gene-silencing mechanisms? As Judd Rice and David Allis discuss in an accompanying News and Views, histone methylation operates independently of DNA methylation as a silencing mechanism in flies and S. pombe, but examples of the converse have yet to be found. Could it be that histone methylation controls DNA methylation, or did these processes co-evolve in higher eukaryotes to provide complex genomes with an additional layer of gene regulation? No doubt, intensive efforts are now underway to pin down the association between these two processes and to improve our understanding of the role of histones in epigenetic gene regulation.

Jane Alfred

(2) References and links

ORIGINAL RESEARCH PAPER Tamaru, H. \& Selker, E. A histone $\mathrm{H} 3$ methyltransferase controls DNA methylation in Neurospora crassa. Nature 414, 277-283 (2001) FURTHER READING Rice, J. R. \& Allis, C. D. Code of silence. Nature 414, 258-261 (2001) WEB SITE

Eric Selker's laboratory:

http://www.molbio.uoregon.edu/facres/selker.htm 
frequency of a DIA4 variant — which leads to loss of protection against the toxic effects of quinones - differed between populations from China and Papua New Guinea, which were assigned to different clusters. Normally, these populations would have been grouped together as Asians, and this important difference would have been missed.

Although these results might not come as a complete surprise to those who study the complexities of population history, they do provide evidence of shortcomings in current drug trials. They also show how genetic classification might be a valuable tool for the future design of clinical trials that will have benefits both in terms of cost savings and human health.

Mark Patterson

(4) References and links ORIGINAL RESEARCH PAPER Wilson, J. F. et al. Population genetic structure or variable drug response. Nature Genet. 29, 265-269 (2001) WEB SITE

David Goldstein's lab:

http://www.ucl.ac.uk/biology/goldstein/Gold.htm
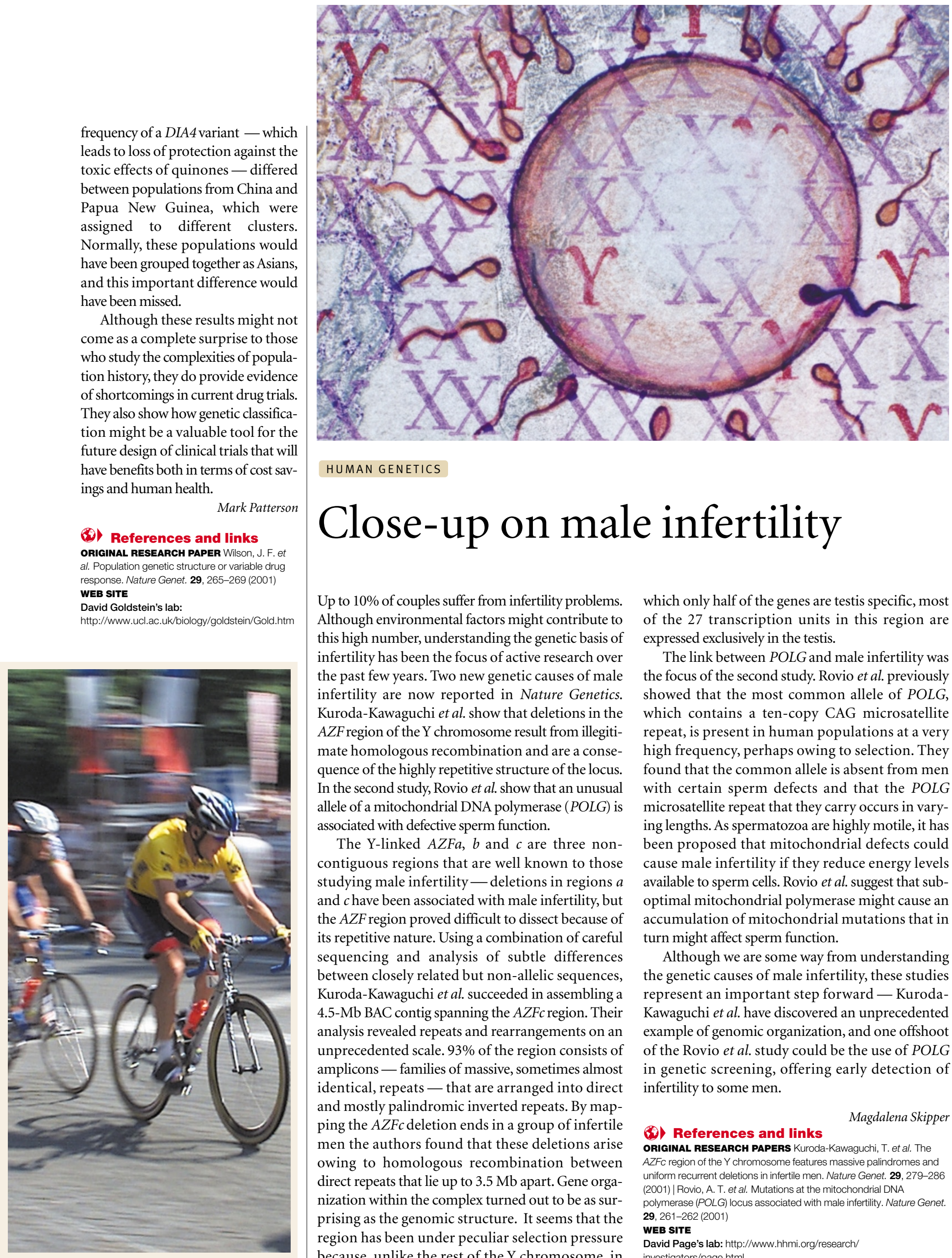

HUMAN GENETICS

\section{Close-up on male infertility}

Up to $10 \%$ of couples suffer from infertility problems. Although environmental factors might contribute to this high number, understanding the genetic basis of infertility has been the focus of active research over the past few years. Two new genetic causes of male infertility are now reported in Nature Genetics. Kuroda-Kawaguchi et al. show that deletions in the $A Z F$ region of the $\mathrm{Y}$ chromosome result from illegitimate homologous recombination and are a consequence of the highly repetitive structure of the locus. In the second study, Rovio et al. show that an unusual allele of a mitochondrial DNA polymerase $(P O L G)$ is associated with defective sperm function.

The Y-linked $A Z F a, b$ and $c$ are three noncontiguous regions that are well known to those studying male infertility - deletions in regions $a$ and $c$ have been associated with male infertility, but the $A Z F$ region proved difficult to dissect because of its repetitive nature. Using a combination of careful sequencing and analysis of subtle differences between closely related but non-allelic sequences, Kuroda-Kawaguchi et al. succeeded in assembling a 4.5-Mb BAC contig spanning the $A Z F c$ region. Their analysis revealed repeats and rearrangements on an unprecedented scale. $93 \%$ of the region consists of amplicons - families of massive, sometimes almost identical, repeats - that are arranged into direct and mostly palindromic inverted repeats. By mapping the $A Z F c$ deletion ends in a group of infertile men the authors found that these deletions arise owing to homologous recombination between direct repeats that lie up to $3.5 \mathrm{Mb}$ apart. Gene organization within the complex turned out to be as surprising as the genomic structure. It seems that the region has been under peculiar selection pressure because, unlike the rest of the $\mathrm{Y}$ chromosome, in which only half of the genes are testis specific, most of the 27 transcription units in this region are expressed exclusively in the testis.

The link between $P O L G$ and male infertility was the focus of the second study. Rovio et al. previously showed that the most common allele of POLG, which contains a ten-copy CAG microsatellite repeat, is present in human populations at a very high frequency, perhaps owing to selection. They found that the common allele is absent from men with certain sperm defects and that the POLG microsatellite repeat that they carry occurs in varying lengths. As spermatozoa are highly motile, it has been proposed that mitochondrial defects could cause male infertility if they reduce energy levels available to sperm cells. Rovio et al. suggest that suboptimal mitochondrial polymerase might cause an accumulation of mitochondrial mutations that in turn might affect sperm function.

Although we are some way from understanding the genetic causes of male infertility, these studies represent an important step forward - KurodaKawaguchi et al. have discovered an unprecedented example of genomic organization, and one offshoot of the Rovio et al. study could be the use of POLG in genetic screening, offering early detection of infertility to some men.

\section{(4) References and links}

Magdalena Skipper

ORIGiNal ReSearch PAPERS Kuroda-Kawaguchi, T. et al. The $A Z F C$ region of the $Y$ chromosome features massive palindromes and uniform recurrent deletions in infertile men. Nature Genet. 29, 279-286 (2001) | Rovio, A. T. et al. Mutations at the mitochondrial DNA polymerase $(P O L G)$ locus associated with male infertility. Nature Genet. 29, 261-262 (2001)

\section{WEB SITE}

David Page's lab: http://www.hhmi.org/research/

investigators/page.html 